\title{
INFLUENCIA DE LA SINTOMATOLOGÍA DEPRESIVA EN LA ADHERENCIA AL TRATAMIENTO ANTITUBERCULOSO EN PACIENTES DEL HOSPITAL SANTA MARIA DEL SOCORRO DE ICA, 2018
}

\author{
TO DETERMINE IF DEPRESSIVE SYMPTOMATOLOGY INFLUENCES THE \\ ADHERENCE TO ANTITUBERCULOUS TREATMENT IN HSMS-ICA PATIENTS, \\ 2018.
}

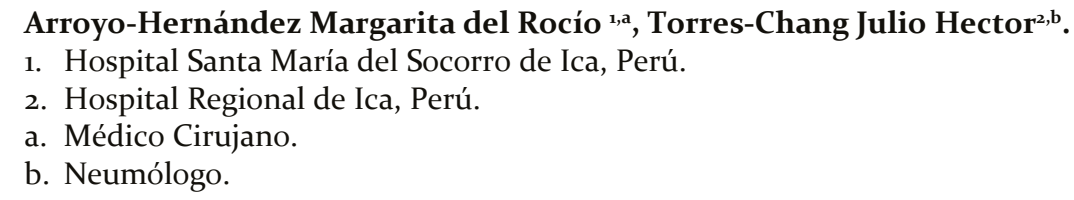

doi: https://doi.org/10.35563/rmp.v8i2.2

\section{Correspondencia:}

Arroyo-Hernández Margarita del Rocío.

Número de celular:

Correo Electrónico:

Mayi_3_94@hotmail.com

\section{Contribuciones De Autoría:}

AHM, TCJ participaron en el diseño del estudio, el análisis de los datos, revisaron críticamente el artículo y aprobaron la versión final.

Conflicto De Intereses: No declarados.

Financiamiento: Autofinanciado.

\section{Como Citar}

Arroyo-Hernández Margarita del Rocío, Torres-Chang Julio Hector. Influencia de la sintomatología depresiva en la adherencia al tratamiento antituberculoso en pacientes del Hospital Santa Maria del Socorro de Ica, 2018. Rev méd panacea. 2019; 8(2): 58-63. doi: https://doi.org/10.35563/rmp.v8i2.270

Recibido: 08 - 03 - 2019

Aceptado: 05 - 05 - 2019

Publicado: 12 - 05 - 2019

\section{RESUMEN}

Objetivo: Determinar la influencia de la sintomatología depresiva en la adherencia al tratamiento antituberculoso en pacientes del Hospital Santa Maria del Socorro de Ica, (HSMSI), 2018. Materiales y métodos: Estudio de tipo transversal, observacional y analítico. La variable independiente fue el nivel de sintomatología depresiva medida con el Test de depresión de Zung; la variable dependiente fue la adherencia al tratamiento recibido mediante el Test de MoriskyGreen, se incluyeron todos los pacientes mayores de 18 años atendidos en la Estrategia Sanitaria de Prevención y Control de Tuberculosis del HSMS registrados en el Libro de seguimiento del año 2018. Resultados: La mediana de edad fue de 30 años, RIQ (21 a 44). El 58,6\% de los pacientes fueron hombres; el, 58,6\% lleva recibiendo más de tres meses tratamiento, el 10,3\% presentaban otras comorbilidades como Diabetes Mellitus y VIH/SIDA; el 17,2\% refieren como habito nocivo consumo de alcohol. El grado de sintomatología depresiva leve fue del $37,9 \%$, seguido de un grado moderado y severo con el $34.5 \%$ y $10,3 \%$ respectivamente. El $65,5 \%$ de los pacientes presentaron mala adherencia al tratamiento. Se encontró que conforme aumenta los grados de sintomatología depresiva la probabilidad de no adherencia al tratamiento se incrementa, sin embargo, no existe asociación significativamente estadística tanto en el análisis crudo como en el ajustado por edad y sexo. Conclusiones: Los grados de manifestación de sintomatología depresiva se incrementan con una mala adherencia al tratamiento antituberculoso en los pacientes atendidos en el Hospital Santa María de Socorro de Ica- 2018.

Palabras clave: Tuberculosis, síntomas depresivos y adherencia al tratamiento.

\section{ABSTRACT}

Objective: To determine if depressive symptomatology influences the adherence to antituberculous treatment in HSMS-Ica patients, 2018. Materials and methods: It is a cross-sectional, observational, analytical and prospective study. The Zung depression test was used to measure the level of depression; while the Morisky-Green Test was used to measure adherence to antituberculous treatment, and the population of patients treated in the Health Strategy for Prevention and Control of Tuberculosis of the HSMS registered in the follow-up book was studied. year 2018, over 18 years. Results: Patients without depressive symptomatology $60 \%$ are adhered and $40 \%$ are not adherent to antituberculous treatment. Among patients with mild depressive symptoms, the highest percentage is $63.6 \%$ and $36.4 \%$ adheres to treatment. Patients with moderate depressive symptomatology $70 \%$ are not adherent to the treatment and $30 \%$ if they are adherent to the treatment. Finally, $100 \%$ of patients with severe depressive symptoms are not adherent to the treatment. As the degree of depressive symptomatology increases, the probability of non-adherence to treatment increases, however, there is no statistically significant association in both the crude analysis and that adjusted for age and sex. $(p=0.425)$. Conclusions: According to the results of the study, depressive symptomatology is associated with poor adherence to antituberculous treatment in patients of Santa Maria de Socorro Hospital in Ica- 2018.

Keywords: Tuberculosis, depressive symptoms, adherence to treatment. 


\section{INTRODUCCIÓN}

La tuberculosis (TB) sigue siendo un problema de salud pública en el Perú (1). Las estrategias de control de la tuberculosis buscan que el paciente no solo cumpla con el tratamiento, sino que este adherido; conocer factores o situaciones que pongan en peligro dicha adherencia será importante para el abordaje de la enfermedad y disminuir su transmisión (2).

La falta de adherencia al tratamiento antituberculoso, no solo es grave porque hace ineficaz el tratamiento, aumentando la morbi-mortalidad, sino que también desde un punto de vista socioeconómico significa un enorme costo para las instituciones de salud y un riesgo para la población en general (3).

Es necesario que los integrantes del equipo de salud de las diferentes estrategias sanitarias de prevención y control de la TB, consideraren no solo dar la medicación al paciente, sino el preocuparse por su integralidad, identificando factores de riesgo que pudieran influenciar en la adherencia al tratamiento, como por ejemplo síntomas depresivos que podrían llevar al abandono o al incumplimiento del mismo (4).

Considerando que el primer pilar para el logro de las metas de la estrategia para acabar con la TB es la "Prevención y atención integral de la TB centrada en el paciente", es necesario hacer un abordaje con un enfoque multidisciplinario y no desatender la esfera mental (5). Por lo mencionado el objetivo de esta investigación busca determinar si la sintomatología depresiva influye en la adherencia al tratamiento antituberculoso en pacientes del HSMSI, 2018.

\section{MATERIALES Y MÉTODOS}

Diseño y población de estudio

Se realizó un estudio de tipo observación, transversal y analítico. Los participantes fueron ñps pacientes atendidos en la Estrategia Sanitaria de Prevención y Control de Tuberculosis del HSMSI registrados en el Libro de seguimiento del año 2018, se seleccionaron a los pacientes con 18 años a más, la muestra fue no probabilístico por conveniencia.

\section{Variables de la investigación}

La variable dependiente fue la adherencia al tratamiento antituberculoso y la variable independiente la presencia de sintomatología depresiva, se consideraron como variables intervinientes la edad y sexo.

\section{Técnicas e instrumentos de investigación}

Se realizó una encuesta autoadministrada, voluntaria y anónima que contenida información con antecedentes sobre enfermedades, hábitos nocivos y el Test de depresión de Zung que informa sobre la presencia y severidad de la sintomatología depresiva, y es sensible a los cambios en el estado clínico, está formado po 20 preguntas relacionadas con la depresión, formuladas la mitad (10 preguntas) en términos positivos y la otra mitad (10 preguntas) en términos negativos y se encuentra dividido por ocho preguntas que miden los síntomas somáticos y otras ocho que miden los síntomas cognitivos, completándose la escala con dos preguntas referentes al estado de ánimo y los últimos dos a síntomas psicomotores (6). La puntuación total no correlaciona significativamente con edad, sexo, estado civil, nivel educacional, económico ni inteligencia (6), esta escala tiene una sensibilidad de un $97 \%$, con una especificidad de $63 \%$ y tiene un $82 \%$ de acierto para discriminar depresión. Sin embargo, no se ha validado con certeza su capacidad para predecir alteraciones somáticas autonómicas. Los índices de fiabilidad son buenos (índices de 0,70-0,80 en la fiabilidad dos mitades, índice a de Cronbach entre 0,79 y 0,92) (7).

La encuesta incluyó además el Test de Morisky-Green para valorar el cumplimiento de la medicación en pacientes con hipertensión arterial (HTA) y usado en la valoración del cumplimiento terapéutico en diferentes enfermedades. Consiste en 4 preguntas con respuesta dicotómica (sí o no), que refleja la conducta del enfermo respecto al cumplimiento (8).

\section{Análisis estadístico}

Los datos fueron procesados mediante análisis descriptivos con frecuencias absolutas y relativas para variables categóricas y mediante la media y desviación estándar o mediana y rango intercuartílico para variables numéricas, previa comprobación de distribución de normalidad con la prueba de Shapiro Wilk. Para calcular las razones de prevalencias (RP) crudas y ajustadas (RPa) con intervalos de confianza al 95\% (IC 95\%) se utilizó un modelo regresión de Poisson, simple y ajustado con varianza robusta. Los análisis se realizaron en el programa Stata v.14.0.

\section{Consideraciones éticas}

El estudio tuvo la aprobación para su ejecución por las autoridades de las instituciones involucradas. Se aplicó un consentimiento informado previa aceptación de los pacientes y no se excluyeron menores de edad. La información recolectada fue anónima.

\section{RESULTADOS}

La mediana de la edad fue de 30 años, con un rango intercuartílico de 21 a 44 años. El 58,6\% de los pacientes fueron hombres y el $65,5 \%$ solteros; el, 58,6\% lleva recibiendo más de 3 meses tratamiento, el 10,3\% presentaban otras comorbilidades como Diabetes Mellitus y VIH/SIDA; el 17,2\% refieren como habito nocivo consumo de alcohol y el $44,9 \%$ no trabajan Tabla 1. 
Tabla 1: Caracteristicas de los pacientes

\begin{tabular}{lcc} 
& $\mathbf{n}$ & $\%$ \\
Edad & 30 & RIQ: $21-44$ \\
Sexo & & \\
Masculino & 17 & 58.6 \\
Femenino & 12 & 41.4 \\
Estado civil & & \\
Soltero & 19 & 65.5 \\
Casado & 4 & 13.8 \\
Conviviente & 6 & 20.7 \\
Tiempo de tratamiento & & \\
Tres meses a menos & 12 & 41.4 \\
Mayor de tres meses & 17 & 58.6 \\
Ocupación & & \\
No trabajan & 13 & 44.9 \\
Trabajan & 16 & 55.1 \\
Comorbilidades & & \\
Hipertensión & 1 & 3.5 \\
Asma & 1 & 3.5 \\
Diabetes Mellitus & 3 & 10.3 \\
VIH/SIDA & 3 & 10.3 \\
Habitos nocivos & & \\
Consumo de alcohol & 5 & 17.2 \\
Drogas & 1 & 3.5 \\
\hline
\end{tabular}

La sintomatología depresiva se presentó en más de la mitad de los pacientes que recibieron tratamiento antituberculoso. El grado de sintomatología depresiva leve es la de mayor proporción en los pacientes (37,9\%), seguida de un grado moderada y severa con el $34.5 \%$ y $10,3 \%$ respectivamente. La adherencia de tratamiento antituberculoso es mala en el $65,5 \%$ de los pacientes que recibieron tratamiento antituberculoso Tabla 3.

Tabla 2: Sexo según sintomatología depesiva y adherencia al tratamiento

\begin{tabular}{lccccc} 
& \multicolumn{2}{c}{ Hombres } & \multicolumn{2}{c}{ Mujeres } & Valor \\
& $\mathbf{n}$ & $\%$ & $\mathbf{n}$ & $\%$ & de $\mathbf{p}$ \\
\hline Nivel de sintomatología depresiva* & & & & & \\
Ninguna & 2 & 40 & 3 & 60 & 0.299 \\
Leve & 5 & 45.5 & 6 & 54.5 & \\
Moderada & 7 & 70 & 3 & 30 & \\
Severa & 3 & 100 & 0 & 0 & \\
$\begin{array}{l}\text { Adherencia a tratamiento** } \\
\text { Adherido }\end{array}$ & 5 & 50 & 5 & 50 & 0.494 \\
No adherido & 12 & 63.2 & 7 & 36.8 & \\
\hline
\end{tabular}

*Test exacto de Fisher, ** Chi cudrado de pearson

Según el sexo, los hombres tuvieron una mayor proporción para presentar un incremento en el grado de sintomatología depresiva, aunque sin diferencia estadística con las mujeres $(P=0 ; 299)$, tampoco se encontró diferencias en el sexo y la adherencia al tratamiento $(P=0,494)$. Asimismo, los pacientes con 30 años a más presentaron un incremento en el grado de sintomatología depresiva, aunque sin diferencia estadística con los pacientes menores de 30 años $(P=1,00)$, tampoco se encontró diferencias entre los grupos de edad y la adherencia al tratamiento $(P=0.359)$ Tabla 3. 
Tabla 3: Grupos de edad según sintomatología depresiva y adherencia al tratamiento

\begin{tabular}{|c|c|c|c|c|c|}
\hline & \multicolumn{2}{|c|}{$<30$ años } & \multicolumn{2}{|c|}{$\geq 30$ años } & \multirow{2}{*}{$\begin{array}{l}\text { Valor } \\
\text { de } p\end{array}$} \\
\hline & $\mathbf{n}$ & $\%$ & $n$ & $\%$ & \\
\hline \multicolumn{6}{|c|}{ Nivel de sintomatología depresiva* } \\
\hline Ninguna & 3 & 60 & 2 & 40 & 1,00 \\
\hline Leve & 5 & 45.5 & 6 & 54.5 & \\
\hline Moderada & 5 & 50 & 5 & 50 & \\
\hline Severa & 1 & 33.3 & 2 & 66.7 & \\
\hline \multicolumn{6}{|c|}{ Adherencia a tratamiento** } \\
\hline Adherido & 6 & 60 & 4 & 40 & 0.359 \\
\hline No adherido & 8 & 42.1 & 11 & 57.9 & \\
\hline
\end{tabular}

${ }^{\star}$ Test exacto de Fisher, ${ }^{* *}$ Chi cudrado de pearson

Se encontró que conforme aumenta los niveles de sintomatología depresiva la probabilidad de no adherencia al tratamiento se incrementa. Sin embargo, no existe asociación estadística tanto en el análisis crudo como en el ajustado por edad y sexo Tabla 4.

Tabla 4: Influencia de la sintomatología depresiva en la adherencia al tratamiento antitubercoloso en pacientes del Hospital Santa María del Socorro de Ica 201

\begin{tabular}{|c|c|c|c|c|c|c|c|c|c|c|}
\hline & \multicolumn{2}{|c|}{ Adherido } & \multicolumn{2}{|c|}{ No Adherido } & \multicolumn{3}{|c|}{ Análisis crudo } & \multicolumn{3}{|c|}{ Análisis ajustado* } \\
\hline & $\mathbf{n}$ & $\%$ & $\mathbf{n}$ & $\%$ & $\mathbf{R P}$ & IC $95 \%$ & Valor p & $\mathbf{R P a}$ & IC $95 \%$ & Valor $p$ \\
\hline \multicolumn{11}{|c|}{ Nivel de sintomatología depresiva } \\
\hline Ninguna & 3 & 60 & 2 & 40 & 1 & & & 1 & & \\
\hline Leve & 4 & 36.4 & 7 & 63.6 & 1.59 & 0.48 a 5.19 & 0.442 & 1.6 & 0.52 a 4.84 & 0.406 \\
\hline Moderada & 3 & 30 & 7 & 70 & 1.75 & 0.54 a 5.62 & 0.348 & 1.64 & 0.55 a 4.91 & 0.371 \\
\hline Severa & 0 & 0 & 3 & 100 & 2.5 & 0.83 a 7.45 & 0.1 & 2.39 & 0.85 a 6.74 & 0.097 \\
\hline
\end{tabular}

${ }^{*}$ Ajustado por edad y sexo

\section{DISCUSIÓN}

En el presente estudio muestra que un mayor nivel en la presentación de sintomatología depresiva se incrementa con una mala adherencia al tratamiento en pacientes con tuberculosis. Si bien no se encontró una asolación estadística, debido a factores relacionados al diseño transversal de estudio y un escaso tamaño de muestra, el riesgo en este grupo de pacientes existe.

Diversos estudios han confirmado que existen factores psicosociales que complican la adherencia a medicamentos y hacen hincapié en la importancia de la atención a la salud mental para garantizar resultados positivos del tratamiento (9). Yan S. y Col (10) (2018); en su estudio de corte transversal coinciden también que los síntomas depresivos mostraron una mayor probabilidad de tener una baja adherencia al tratamiento que aquellos sin síntomas.

Este estudio coincide también con el estudio tipo casos y controles realizado por Petit y col.(11) cuya conclusión fue que la falta de adherencia al tratamiento relacionado con la depresión, incrementa cuatro veces más el riesgo de culminación del tratamiento antituberculoso. Otro estudio internacional realizado por Plata-Casas (12) en el 2015 coincide en que factores psico sociales que pudieran influir en el paciente, contribuyen al incumplimiento y abandono del tratamiento.

Respecto a estudios realizados en Perú, Morales-Miranda (2015) (13) buscó determinar si la depresión influía en la adherencia al tratamiento en los pacientes multidrogorresistentes de la Red Asistencial Almenara en EsSalud-Lima, encontrando asociación entre depresión y adherencia al tratamiento, siendo en este estudio significativamente estadística. Maldonado-Alvarado (14) (2016), en un estudio con las mismas variables realizado en Pucallpa durante los años 2014-2015, encuentra asociación significativamente estadística entre la depresión y la adherencia al tratamiento. Riquero (15) (2012) en un estudio de tipo comparativo no experimental, concluye también en una 
asociación entre la depresión y la mala adherencia al tratamiento.

La tuberculosis continúa es un problema de salud pública que hace poner en acción diferentes estrategias no solo de prevención sino también de control, por las consecuencias que esta trae. Sin embargo, a pesar de todos estos esfuerzos no se ha logrado erradicar su impacto en la población. Un reto para el control es conseguir que las personas que se diagnostican e inician el tratamiento persistan hasta terminarlo para así lograr niveles óptimos de curación.

En conclusión, los niveles de sintomatología depresiva se incrementan con la mala adherencia al tratamiento antituberculoso en pacientes del Hospital Santa María del Socorro en el año 2018

Se debe recomienda insistir con las políticas del MINSA en la cual el paciente debe acudir al departamento de Psicología los primeros meses de su tratamiento, como parte de su enfoque multidisciplinario. Es innegable que el diagnóstico de la tuberculosis trae consigo la presencia de sintomatología depresiva; por lo cual se recomienda no solo orientación psicológica al paciente, sino también a su familia ya que su apoyo hará que el paciente se involucre mejor con su enfermedad, mejorando la adherencia al tratamiento. Se debe de continuar con las capacitaciones al personal de salud, para detectar a tiempo sintomatología depresiva y así abordarlos a tiempo, evitando que se interponga en la adherencia al tratamiento.

La estrategia DOTS (Sistema de Tratamiento por Observación Directa) sigue siendo el núcleo de la estrategia Alto a la TB para combatirla y aumentar la adherencia al tratamiento; sin embargo, en el presente estudio se evidencia la mayor proporción de la población estudiada, no cuenta con una adecuada adherencia; por lo cual se recomienda aumentar las sesiones educativas acerca de la importancia del cumplimiento del tratamiento de la tuberculosis no solo en que la medicación se consuma diariamente, sino también en un horario constante y establecido. 


\section{REFERENCIAS BIBLIOGRÁFICAS}

1. Ministerio de Salud del Perú. Plan de Intervención de Prevención y Control de Tuberculosis en Lima Metropolitana y regiones priorizadas de Callao, Ica, La Libertad y Loreto, 2018-2020; Documento técnico;2018

2. Yagui Moscoso M, Jave HO, Curisinche Rojas $M$, Gutiérrez C, Romaní Romaní F. Agenda Nacional de Investigación en Tuberculosis en Perú, 2011-2014. Rev Panam Salud Publica. 2013;33(1):151-8.

3. Ministerio de Salud del Peru. Plan de Intervención de Prevención y Control de Tuberculosis en Lima Metropolitana y regiones priorizadas de Callao, Ica, La Libertad y Loreto, 2018-2020; Documento técnico;2018

4. Rios J. Situación de Tuberculosis en el Perú y la respuesta del Estado (Plan de Intervención, Plan de Acción) Estrategia de Prevención y Control de TB-DPCTB DGIESP-MINSA; 2017

5. Aplicación de la estrategia fin de la TB: aspectos esenciales. Ginebra: Organización Mundial de la Salud; 2016.

6. Vélez, C Barrera, AV Benito, M Figueroa, SM Franco. Estudio de síntomas depresivos mediante la Escala de autoaplicación de Zung en varones privados de la libertad de una ciudad de Colombia Rev Esp Sanid Penit 2016; 18: 43-48

7. Lezama S. Propiedades psicométricas de la escala de Zung para síntomas depresivos en población adolescente escolarizada colombiana. Psicología. Avances de la disciplina. 2012, 6: 28-32

8. M.Á. Rodríguez Chamorro y col. Herramientas para identificar el incumplimiento farmacoterapéutico. Pharmaceutical Care España 2013; 11(4): 183-191
9. Argiro Pachi, Dionisios Bratis, Georgios Moussas, and Athanasios Tselebis, "Psychiatric Morbidity and Other Factors Affecting Treatment Adherence in Pulmonary Tuberculosis Patients,. Tuberculosis Research and Treatment, vol. 2013

10. Yan S. y col. Nonadherence to Antituberculosis Medications: The Impact of Stigma and Depressive Symptoms. Am J Trop Med Hyg. 2018 Jan;98(1):262-265.

11. Pettit AC, Cummins J, Kaltenbach LA, Sterling TR, Warkentin JV. Non-adherence and drug-related interruptions are risk factors for delays in completion of treatment for tuberculosis. Int J Tuberc pulmón Dis. 2013 abr; 17 (4): 486-92

12. Plata-Casas LI. Factores asociados a la no adherencia al tratamiento anti tuberculosis. Rev. cienc. cuidad. 2015; 12(2): 26-38

13. Morales-Miranda E. Influencia de la depresión sobre la adherencia al tratamiento en pacientes TB-MDR de la Red Almenara de Essalud.Lima.2015. Universidad Ricardo Palma. Lima-Peru 2015

14. Maldonado-Alvarado A. y col. Influencia de la depresión sobre la adherencia al tratamiento en pacientes con TB-MDR en el Hospital Regional de Pucallpa en el periodo enero 2014- diciembre 2015. Univerisad Nacional de Ucayali. Pucallpa-Peru 2016

15. Riquero, P. (2014). Depresión en pacientes con tuberculosis según tipo de tratamiento de la red urbana del Minsa Chiclayo, 2012 Tesis de pregrado, Universidad Católica Santo Toribio de Mogrovejo, Chiclayo, Perú.
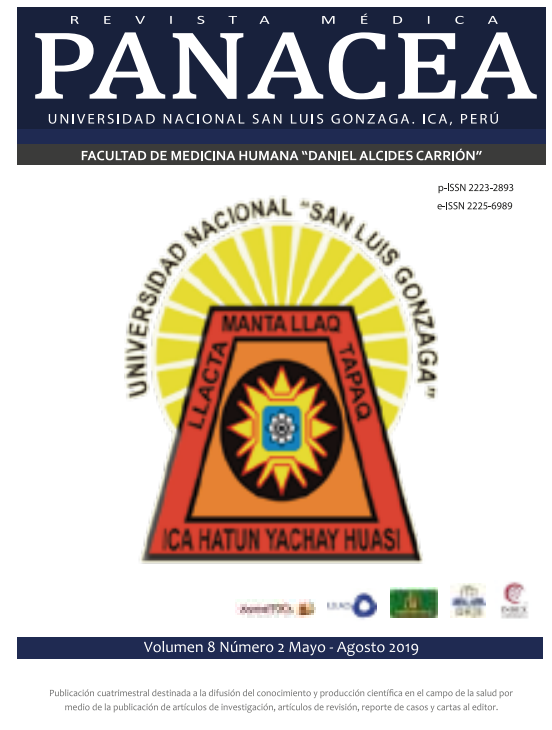